\title{
La ley como discurso \\ Alcances teóricos y empiricos de la norma legal como instrumento ideológico en la construcción de representaciones sociales ${ }^{1}$
}

\author{
Gonzalo Albornoz Barra \\ Universidad de la Frontera de Temuco
}

Fecha de recepción 12-08-2018

Fecha de aceptación 11-10-2018

\section{RESUMEN}

La Ley vista como discurso, permite la reflexión sobre sus alcances más allá de los efectos directos de su aplicación en actores involucrados con procesos de judicialización. Como discurso, y como instrumento cargado de legitimidad, la Ley permite el desplazamiento de ideologías, a la vez que se transforma en un instrumento cargado de una falsa veracidad para la construcción de representaciones sobre los fenómenos que regula. Estos alcances de la Ley, se dan mediante distintas formas de uso, y a través de distintas plataformas consumo que operan en la cotidianidad de los actores.

PALABRAS CLAVE: Ley, discurso, ideología, representaciones sociales.

\begin{abstract}
The Law, seen as discourse, allows reflection on its scope beyond the direct effects of its application on actors involved in judicialization processes. As discourse, and as an instrument loaded with legitimacy, the Law allows for the displacement of ideologies, while at the same time it becomes an instrument loaded with a false truthfulness for the construction of representations about the phenomena that regulates. These scopes of the Law, are give througth different forms of use, and through different platforms of consume that operate in the everyday life of the actors.
\end{abstract}

KEYWORDS: Law,discourse, ideology, social representations.

$1 \quad$ Artículo realizado en base a los resultados de la investigación titulada: "La ley 18.314 y la idea de criminalidad terrorista para distintos actores en su vida cotidiana" (2018). El estudio citado se hizo bajo la dirección del Dr. Carlos del Valle dentro del proyecto FONDECYT n 1150666 "La construcción del imaginario social de la justicia en los relatos periodísticos publicados por el diario El Mercurio de Chile, entre 1850 y 2014, en contexto del conflicto Estado-nación y pueblo Mapuche: Continuidades y cambios" (2016-2018). 


\section{La Ley como discurso ideológico}

$\mathrm{H}$ ay ciertas premisas a las cuales es preciso introducir antes que todo. Un primer punto es que la construcción sobre la noción de Ley en el presente artículo se realiza mediante enfoques teóricos que hacen suponer, de modo general, que el ejercicio de la Legalidad ${ }^{2}$ se constituye por características y dinámicas que involucran elementos como: a) Contextos particulares que determinan las bases ideológicas de la estructura de la Ley; b) Dinámicas de poder; c) Distintas formas de saber; y d) Ideas construidas y reconstruidas socialmente por los actores a través de usos y consumos culturales en su vida cotidiana en base (Foucault 1978, 1979, 1999; Van Dijk 1994, 2005a, 2005b; Van Dijk y Mendizábal 1999).

Un segundo punto a precisar es que, vista como discurso, la ley — cualquiera sea esta- no solo tiene efectos mediante su aplicación. La ley como discurso se transforma en un instrumento que traspasa los límites de lo estrictamente jurídico, pasando a tener variados efectos en lo social mediante múltiples formas de uso y consumo como: sentencias, artículos académicos, discursos políticos, discursos en la prensa o el propio texto legal entre otros. Así, la ley no es solo un instrumento jurídico, y mucho menos está desprovista de subjetividades, también es una herramienta que expresa y permite el tránsito de ideas sobre los fenómenos que regula (Foucault 1978, 1979, 1999; Nieto 2007).

Un tercer aspecto es que la Ley no es un discurso común, sino más bien un discurso cargado de poder e ideologías (Aguilera y González 2011; Althusser 1989; Foucault 1978, 1979, 1999, 2013; Nieto 2007; Van Dijk 1994, 2005a, 2005b). La ley es un discurso legitimado institucional y socialmente mediante una serie de procesos que se dan en una suerte de objetivación de subjetividades, un discurso al parecer "carente de implicaciones valorativas" (Vilajosana 2010:11) que faculta su uso como instrumento apropiado para definir conductas e implicancia de las mismas.

Resumiendo, la investigación partió de una premisa general que Santiago Nieto en Estudios en homenaje a Marcia Muñoz de Alba Medrano resume de forma precisa cuando dice que:

El Derecho no es una disciplina pura. Todo proceso que tenga que ver con la producción de normas generales, con la aplicación de estas a casos concretos, con la enseñanza y la investigación jurídica, tiene una profunda carga ideológica. Cada operador jurídico, de conformidad con sus experiencias y convicciones personales,

2 Se hace referencia a la legalidad como el conjunto de prácticas e instrumentos involucrados en las Representaciones sociales sobre lo justo; $y$ en tanto, sobre el carácter legítimo de la Ley y las prácticas judiciales para regular la vida social. 
e incluso, de acuerdo con su propia posición epistemológica, imprime a las diversas actividades del ámbito jurídico una determinada concepción del Derecho (Nieto 2007: 387).

El vasto trabajo de Michel Foucault en La verdad y las formas jurídicas, entre tantos otros trabajos y clases magistrales al respecto, también entrega insumos importantes a esta noción de legalidad cuando dice que las prácticas judiciales: "son algunas de las formas empleadas por nuestra sociedad para definir tipos de subjetividad, formas de saber $y$, en consecuencia, relaciones entre el hombre y la verdad que merecen ser estudiadas" (Foucault 1978: 5).

Con todo, partiendo de la base que ley es un discurso ideológico legitimado socialmente, surgió la necesidad de explorar cómo las ideologías de la Ley Antiterrorista chilena podían tener algún grado de presencia en la construcción de ideas sobre el fenómeno de la criminalidad terrorista.

\section{La ideología y la representación social como elementos para la construcción de subjetividades en la cotidianidad}

Establecer diálogos entre los postulados de Foucault, los términos en los que Van Dijk define ideología y la teoría de las representaciones sociales propuesta por Berger y Luckmann no es tarea fácil, principalmente dada la resistencia de Foucault a la idea de representación social de la realidad, o a la noción dialéctica de ideología. No obstante lo anterior, se pueden extraer algunos elementos que permiten avanzar en la línea propuesta destacando algunos puntos de conexión entre los autores.

En primer lugar, se aprecia como es que aparece la noción de representación como un "conjunto de ideas" en todos los autores mencionados (Araya 2002; Berger y Luckmann 1991; Jodelet 2008; Van Dijk 2005a, 2005b). En este sentido, representar es establecer un equivalente sobre algo; dicho de otra forma, se establece una representación cuando sobre un objeto o fenómeno " $\mathrm{A}$ ", se establece otro similar "B" que permite mediar la comprensión del primer objeto o fenómeno (Araya 2002). Así, como representación social se debe entender este mismo proceso de equivalencia, pero a un nivel macro:

Las personas conocen la realidad que les circunda mediante explicaciones que extraen de los procesos de comunicación y del pensamiento social. Las representaciones sociales, sintetizan dichas explicaciones y en consecuencia, hacen 
referencia a un tipo específico de conocimiento que juega un papel crucial sobre cómo la gente piensa y organiza su vida cotidiana: el conocimiento del sentido común (Araya 2002: 11).

En concreto, se puede entender a las representaciones sociales como un punto de conexión entre lo psicológico y lo social; o como menciona Jodelet en La representación social - Fenómeno, concepto y teoría:

[...] una forma de conocimiento específico, el saber de sentido común, cuyos contenidos manifiestan la operación de procesos generativos y funcionales socialmente caracterizados. En sentido más amplio, designa una forma de pensamiento social. Las representaciones sociales constituyen modalidades de pensamiento práctico orientados hacia la comunicación, la comprensión y el dominio del entorno social, material e ideal (Jodelet 1986: 474).

En este contexto, el rol de las ideologías, así como de la cultura, los códigos o los valores, se encuentran en el contexto particular de cada sujeto y grupo. Estos, como una forma de interpretar y pensar la realidad cotidiana, despliegan su actividad mental para fijar una posición respecto de situaciones, acontecimientos, objetos o relaciones (Jodelet 1986).

Luego, se da otro punto en común en la importancia que tiene el devenir histórico particular y social en la construcción de subjetividades. Al respecto, es importante considerar que las interpretaciones sobre el mundo guardan estrecha relación con las particularidades históricas y culturales de los sujetos y los grupos, las cuales también influyen en la construcción de ideologías. Foucault, aunque ajeno a las nociones de representación social e ideología, se remite a considerarlas en alguno de sus trabajos:

[...] no se puede dejar de advertir que cuando Foucault trata específicamente del sujeto, como es el caso en el curso del Colegio de Francia "Hermenéutica del sujeto" (2001a), recurre a la noción de representación como "conjunto de ideas". Esta obra es la única cuyo índice contiene el término"representación", mencionado dieciocho veces. Los pasajes donde se utiliza este término ponen de manifiesto que la representación interviene en el proceso de subjetivación como objeto de un trabajo de reflexión y de elección, en el marco de las técnicas del sí mismo y del conocimiento de sí mismo practicadas en la Antigüedad. Más aún, este trabajo sobre las representaciones se realiza bajo la orientación de un director de conciencia, lo que remite al papel que desempeña la interacción en la elaboración de la representación de sí mismo (Jodelet 2008: 42). 
Con todo, Ideología y Representación son términos confluyentes. Van Dijk sostiene que las ideologías son un sistema de creencias fundamental, que organiza y fundamenta las representaciones sociales compartidas por los miembros de grupos ideológicos (2005a: 12; 2005b: 18-20). Visto desde esta perspectiva, la ideología como forma de representación de grupos, encaja con las formas en que se construye la legitimidad de la ley en las propuestas de Foucault.

El saber y la verdad responden a prácticas de poder. Bajo estas prácticas, y erróneamente, la ley y la legalidad se transforman en instrumentos y ejercicios representados socialmente como legítimos, y en el mismo sentido, como herramientas desprovistas de subjetividad, lo que las valida como prácticas de saber y verdad para el uso cotidiano.

Bajo esta idea, la ley como discurso podría desplazar ideologías y representaciones, a la vez que construir narrativas culturales sobre los fenómenos que regula. Estos desplazamientos, tendrían lugar bajo distintas formas de uso y consumo de la ley como: la propia norma legal, sentencias, trabajos y conferencias académicas, discursos políticos, artículos de prensa, titulares o simples conversaciones de carácter informal en donde se utilice la ley como instrumento de saber y/o verdad en la comprensión del fenómeno tratado.

Michel De Certeau (1986) señala que los actores producen y reproducen interpretaciones sobre el mundo a través de, básicamente, tres procesos: a) El consumo en su amplia definición; b) La producción propia de cada actor basado en lo consumido; y c) La reproducción de aquellas producciones propias, que a la vez dan paso a nuevas prácticas de consumo para otros sujetos o comunidades.

Así, en la dinámica denominada "producción de los consumidores" (De Certeau, 1986), las ideologías y representaciones que constituyen la construcción de una ley, podrían tener algún grado de presencia en las interpretaciones que los sujetos hagan en el ámbito de la cotidianidad; esto, siempre determinado por los diferentes tipos de uso y consumo del discurso o la norma legal.

\section{Alcances empíricos de la ley como discurso ideológico en la producción de representaciones sociales. El caso de la Ley Antiterrorista chilena}

Ver la ley como un discurso ideológico que sirve como base legítima en la construcción de representaciones sociales no solo tiene alcances teóricos. En efecto, y a la luz de un estudio reciente titulado: La ley 18.314, y la idea de criminalidad terrorista para distintos actores en su vida cotidiana (Albornoz 2018), los des- 
plazamientos que generan los distintos tipos de uso de la norma aludida, pueden servir de ejemplo a la hora de establecer los efectos empíricos que tiene la Ley como discurso ideológico en la construcción de Representaciones sociales.

Se debe tener en cuenta que las representaciones e ideologías que sustentan la Ley Antiterrorista chilena estuvieron en gran medida determinadas por la presión mediática generada por una huelga de hambre de 38 comuneros mapuche ${ }^{3}$ que exigían la modificación de la ley en cuestión; lo que finalmente desemboca en la reforma de Ley $n^{\circ} 20.467$ (2010).

En concreto, los cambios que supuso la reforma del año 2010 a la Ley Antiterrorista chilena se basaron en la modificación de las penas asociadas al delito de incendio, junto con otros cambios como la restricción de aplicabilidad a menores de 18 años y otros reordenamientos de tipo penal. Dichos cambios, van en la línea de lo exigido por los comuneros en huelga, ya que los cambios antes mencionados permitían su juicio sin el agravante de dolo terrorista (lo que hacía bajar considerablemente su sentencia).

Así, los discursos que dieron forma a la discusión sobre terrorismo en la ley, transcurrieron principalmente entre los modos estratégicos de enfrentar el conflicto coyuntural de la huelga a través de una reforma, y a la señal positiva que daría la modificación de la ley al pueblo Mapuche a propósito de las demandas históricas. Con esto, la discusión sobre los elementos constitutivos y característicos del fenómeno de la criminalidad terrorista se desplazan a un plano menor, a la vez que se comienzan a construir asociaciones simbólicas entre las ideas de "terrorismo" y el pueblo Mapuche.

Cabe mencionar que la criminalidad terrorista tiene, en general y según diversos actores, tres características principales: a) Se manifiesta a través de grupos considerados como una otredad; b) que persiguen un fin político e ideológico; c) en pugna con un Estado.

Los usos de la ley antiterrorista como discurso en las producción y reproducción de definiciones, opiniones, creencias o estereotipos sobre el fenómeno de la criminalidad terrorista, se dan bajo distintas plataformas de consumo. En estas destacan principalmente: el uso de redes sociales (principalmente grupos cerrados en la aplicación "WhatsApp"), conversaciones de tipo informal en el contexto laboral o familiar de los actores, consumo de prensa escrita, radial y televisiva, documentos jurídicos, documentos y conferencias académicas y medios digitales alternativos.

3 Caso Hector Llaitul y otros. 
Es importante considerar que cada plataforma responde directamente al rol particular del actor. De esta forma, las representaciones que construyen socialmente el fenómeno de la criminalidad terrorista se ven determinadas por los ámbitos de emergencia de cada discurso. Así, la reproducción de ideas sobre terrorismo, y el uso de la ley bajo distintas formas discursivas se genera y circula de forma diferenciada según cada comunidad interpretativa (Fish 1989, 1995).

Con todo, el hecho de que los ejes principales de la discusión parlamentaria se giren en torno a generar modos estratégicos de solución coyuntural a la huelga de hambre, a una asociación entre el fenómeno del terrorismo y las demandas históricas del pueblo Mapuche, y considerando que la aplicación de esta ley se ha dado con forma sostenida en la Araucanía y a personas del pueblo Mapuche; la "Producción de los consumidores" que acontece a raíz de las diversas formas de uso de la Ley como discurso, permiten el desplazamiento de ideologías y representaciones sobre el fenómeno del terrorismo. En estas representaciones, la Ley Antiterrorista aparece como un elemento de incuestionable veracidad conceptual para definir los límites de la criminalidad terrorista; no obstante su uso procedimental sea cuestionado por casi todos los sujetos que formaron parte del estudio, y en virtud de que, en efecto, las discusiones parlamentarias no se concentraron jamás en la definición de límites conceptuales que ayuden a diferenciar y caracterizar un acto terrorista de otro tipo de delito.

Luego, el desplazamientos de la categoría simbólica de Mapuche terrorista, se funda en las características con las que se construye la representación social de terrorismo. Aquí, el Mapuche aparece como aquel grupo cultural que reúne las características principales de este tipo de actos: a) como un otro ideológicamente distinto; b) que persigue el fin político de una recuperación de territorio ancestral; c) en pugna con el Estado.

Las ideologías y representaciones que constituyen una ley, se desplazan como categorías legítimas de uso y consumo social en distintas plataformas de emergencia discursiva. Esta legitimidad de la que gozan los ejercicios legales, generan un uso y consumo ausente de reflexión crítica, lo que hace más sencillo el ejercicio de procesos criminalizadores mediante la aplicación de normas como la Ley Antiterrorista en Chile.

Al respecto, Gregor Bruce (2013: 27) en Definition of terrorism social and political effects aporta una frase a lo menos interesante cuando dice que:

The legal profession desires a definition that can be used for the successful prosecution and conviction of accused terrorists. Defence or an appeal by an 
accused terrorist is easier if the crimes are ambiguously defined [La profesión jurídica desea definiciones que puedan utilizarse para el enjuiciamiento y la condena exitosa de los acusados de terrorismo. La defensa o la apelación de un terrorista acusado es más fácil si los crímenes son definidos de forma ambigua].

En efecto, el caso chileno de la Ley Antiterrorista evidencia cómo la ambigüedad de la norma permite que se desarrollen procesos de criminalización mediante una aplicación sostenida a personas de origen mapuche en una zona particular del país, la Araucanía. Esta criminalización, encuentra apoyo en la producción y reproducción de ideologías y representaciones sociales sobre el fenómeno de la criminalidad terrorista, y sobre la ley como instrumento legítimo.

Así, las características del conflicto histórico Mapuche contra el Estado chileno, permiten la construcción de representaciones del mapuche como un enemigo íntimo (Del Valle 2018, Jakobs y Cancio 2003, Nandy 1983). Lo anterior, debe ir sumado a que la discusión parlamentaria gira en torno a la presión mediática generada por la huelga de hambre de los 38 comuneros, y a que los objetivos que perseguía la reforma era dar con modos políticos y estratégicos para la solución de aquel problema mediático en el contexto del conflicto histórico mencionado.

Finalmente, y dado el contexto de emergencia discursiva antes descrito, la Ley Antiterrorista chilena habla sobre mapuches, sobre un conflicto histórico e ideológico, y sobre una zona particular donde se ejerce el terrorismo (la Araucanía). Con esto, se construyen narrativas respecto del fenómeno del terrorismo en Chile, mientras que la aplicación de la norma da paso a producciones y reproducciones de estas representaciones sobre la criminalidad terrorista en las distintas plataformas de uso y consumo de la ley.

Con todo, los efectos de la ley traspasan el ámbito jurídico, teniendo también impacto en lo social como herramientas de uso y consumo conceptual cubierto de legitimidad para la producción y reproducción de verdades y saberes que, posteriormente, darán paso a la construcción de ideologías y representaciones sobre los fenómenos que esta regula. 


\section{Bibliografía}

AgUILERA, Rafael y GonZÁLEZ, Joaquín (2011). “Derecho, Verdad y Poder en la teoría políticojurídica de Michel Foucault". Revista A Parte Rei. España.

ALBORNOZ, Gonzalo (2018). "La ley 18.314, y la idea de criminalidad terrorista para distintos actores en su vida cotidiana: expresiones desde la vida cotidiana, de las relaciones entre la construcción ideológica de la ley antiterrorista entendida como discurso, y las representaciones sociales sobre la criminalidad terrorista desde la perspectiva de diversos actores en la Araucanía" (Tesis de maestría). Universidad de la Frontera. Temuco, Chile. ALTHUSSER, Louis (1989). Ideología y aparatos ideológicos del Estado. México: Editorial Siglo XXI. BENGOA, José (2001). Historia de un conflicto: El Estado y los Mapuches en el siglo XX. Chile: Editorial Planeta.

BERGER, Peter y LUCKMANn, Thomas (1991). La construcción social de la realidad. Buenos Aires: Amorrortu editores.

BiBLIOTECA DEL CONGRESO NACIONAL (2018). Evolución de la legislación antiterrorista en Chile y España. Valparaíso, Chile: Departamento de estudios, extensión y publicaciones.

BRUCE, Gregor (2013): "Definition of terrorism social and political effects". Journal of Military and Veterans' Health. Vol. 21 Num. 2. Australia.

DE CERTEAU, Michel (1986). La invención de lo cotidiano. I: Las artes del hacer. México: Universidad Iberoamericana.

Del VALLE, Carlos (Enero de 2018). "Las narrativas del proyecto civilizatorio y la producción del enemigo íntimo en la industria cultural chilena: modos y estrategia de sujeción". En M. Loayza (Presidencia). Diacronía de la crisis: conflictos, desigualdades y narrativas en los medios de comunicación en Latinoamérica. Conferencia llevada a cabo en Seminario de la Universidad Nacional Mayor de San Marcos. Lima, Perú.

FISH, Stanley (1995). Is There a Text in this Class? The Authority of Interpretive Communities. Cambridge: Harvard University Press. USA.

FOUCAULT, Michel (1996). La verdad y las formas jurídicas. Barcelona: Editorial Gedisa.

FouCAULT, Michel (2003). La arqueología del saber. Buenos Aires: Editorial Siglo XXI.

Foucault, Michel (2014). Defender la sociedad. Buenos Aires: Fondo de Cultura Económica.

GIL, Juan (1968). “Derecho e ideología”. Revista de Estudios Políticos no 157. España.

GIMÉNEZ, Gilberto (1981). Poder, Estado y discurso. Perspectivas sociológicas y semiológicas del discurso jurídico y político. México: Universidad Nacional Autónoma.

JAKOBS, Gunther y CANCIO, Manuel (2003). Derecho penal del ciudadano y derecho penal del enemigo. Madrid: Editorial Civitas.

JODELET, Denise (2008). “El movimiento de retorno al sujeto y el enfoque de las representaciones sociales". Revista CONNEXION n 89. Francia: Editorial Érès. 
JODELET, Denise (1986). “La representación social: fenómenos, concepto y teoría". En Moscovici, Serge (Comp.), Psicología social Il 469-494. Barcelona: Editorial Paidós.

MiELES, Ernesto (2004).“El concepto de Derecho en Foucault, la Ley y la crítica del paradigma liberal". Revista IUSTA. Conferencia pronunciada en el espacio académico de la Cátedra Fray Bartolomé de las Casas. Colombia.

NIETO, Santiago (2007). Metodología jurídica e ideologías en el derecho. sobre la influencia de la ideología en los ámbitos de aplicación del derecho. Estudios en homenaje a Marcia Muñoz de Alba Medrano. México: Universidad Autónoma de México.

NANDY, Ashis (1983). El enemigo íntimo: pérdida y recuperación del yo bajo el colonialismo. Nueva Delhi: Oxford University Press.

VAN DIJK, Teun (2005a). "Ideología y análisis de discurso". Quórum académico Vol. 2 número 2. Universidad de Zulia, Venezuela.

VAN DIJK, Teun (2005b). "Política, ideología y discurso". Revista Utopía y praxis Latinoamericana. Universidad de Zulia, Venezuela.

VAN DIJK, Teun y MENDIZÁBAL, Ivan (1999). Análisis del discurso social y político. Quito: Serie Pluriminor ABYA-YALA. 